This is a pre-print of an article published in

Annals of Physics, 418 (2020) 168191

The final authenticated version is available online at:

https://doi.org/10.1016/j.aop.2020.168191

\title{
Analyses of Mössbauer experiments in a rotating system: Proper and improper approaches
}

\author{
Alexander L. Kholmetskii, ${ }^{1}$ Tolga $\operatorname{Yarman}^{2}$, O. $\operatorname{Yarman}^{3}$, M. Arik \\ ${ }^{1}$ Department of Physics, Belarus State University, Minsk, Belarus; alkholmetskii@gmail.com \\ ${ }^{2}$ Istanbul Okan University, Istanbul, Turkey \& Savronik, Eskisehir, Turkey \\ ${ }^{3}$ Istanbul University, Istanbul, Turkey \\ ${ }^{4}$ Bogazici University, Istanbul
}

\begin{abstract}
We examine various attempts to interpret the results of modern Mössbauer rotor experiments and show that the most recent approach developed by Podosenov et al. (Ann. Phys., doi.org/10.1016/j.aop.2019.168047) to understand these results implies an experimental configuration which has never been realized in the known experiments on this subject, and thus leaves the problem of the physical interpretation of the observed energy shift between emission and absorption lines in a rotating system under the framework of general relativity open.
\end{abstract}

\section{Introduction}

As is known, the first series of Mössbauer rotor experiments aiming to verify the relativistic time dilation under laboratory conditions had been carried out in the early 60 s soon after the discovery of the Mössbauer effect (see, e.g., [1-6]). The latter effect leads to the following prediction for the relative energy shift between the line of a source of resonant radiation (located on the rotational axis) and the line of a resonant absorber (located at the rotor rim):

$$
\frac{E_{s}-E_{a}}{E_{s}} \equiv \frac{\Delta E}{E}=-\frac{1}{2} \frac{u^{2}}{c^{2}} \text {. }
$$

Here, $E_{s}$ is the energy of the resonant radiation for the source, and $E_{a}$ is the energy of the resonant radiation for the absorber, with $u$ standing for the tangential velocity of the absorber, and $c$ for the light velocity in vacuum (see Fig. 1).

We would like to point out that the "minus" sign on the rhs of eq. (1) corresponds to the blue shift of the energy of the resonant radiation, where $E_{a}>E_{s}$.

All of the early experiments [1-6] reported the confirmation of the relativistic expression (1) with a relative uncertainty near $1 \%$; though, amongst them, only the experiment by Kündig [5] could be considered reliable due to his capability to measure simultaneously the shapes and positions of the resonant line versus the tangential velocities of the absorber $u$. All other experiments of the $20^{\text {th }}$ century [1-4, 6] evaluated only the intensity of the resonant radiation passing across the absorber at different tangential velocities $u$, and were not secured from the influence of rotor vibrations (which are always present) on the measurement results. This assertion is explained by the fact that any vibrations broaden the resonant lines, so that the two effects, i.e., the variation of the detector's count-rate due to the relative energy shift between emission and absorption lines, and the variation of its count-rate due to the broadening of the absorption line caused by vibrations - become indistinguishable from each other at any $u$. Therefore, only the 
experiment by Kündig - where the shift of the absorption line upon the energy scale was measured independently from its broadening - provided reliable data for testing the validity of eq. (1). Kündig actually reported the implementation of eq. (1) with a relative uncertainty of less than $1 \%$, and after the 1960s, Mössbauer rotor experiments were not repeated for about half a century.

Our own motivation to re-analyze these experiments was related to the prediction by $\mathrm{T}$. Yarman [8] for a source at rest and a rotating absorber,

$$
\frac{\Delta E}{E}=-\frac{u^{2}}{c^{2}} \text {. }
$$

For its verification, ${ }^{1}$ we first of all took a closer look at the result of the experiment by Kündig [5]. In this way, we found unfortunate computational errors in Kündig's data processing that led to the following corrected re-estimation of the result of the Kündig experiment [9]:

$$
\frac{\Delta E}{E}=-(0.596 \pm 0.006) \frac{u^{2}}{c^{2}} \text {. }
$$

Putting aside the deviation of eq. (3) from eq. (2), we emphasize that the estimation (3) anyway substantially disagreed with the classical relativistic prediction (1), and due to this reason, it attracted considerable attention from the scientific community, indicating the need to carry out new Mössbauer experiments in a rotating system.

In a general formulation, the goal of such a new experiment is the measurement of the coefficient $k$ in the equation

$$
\frac{\Delta E}{E}=-k \frac{u^{2}}{c^{2}} .
$$

Just before the performance of our own Mössbauer rotor experiments, we kept in mind three possible results: $k=0.5$ (eq. (1)), $k=0.6$ (eq. (3)), $k=1.0$ (eq. (2)). Thus, our own measurements were aimed to select one of these values.

Surprisingly for us, the experiment carried out in Minsk in 2008 [10, 11], and its improved version carried out in Istanbul in $2014[12,13]$, confirmed none of these predictions but rather disclosed the equality $k=2 / 3 .^{2}$

In these experiments, we did not repeat the approach by Kündig, who based himself on a linear Doppler modulation of the energy of the emitted resonant $\gamma$-quanta, because some unaccounted-for systematic errors in the evaluation of the coefficient $k$ in eq. (4) do inevitably emerge (see, e.g. [11]). Thus, we did not try to repeat directly the measurement scheme by Kündig, but followed the experimental scheme used in $[1-4,6]$, where the source of resonant radiation is rigidly fixed on the rotor axis. At the same time, unlike the experiments [1-4, 6], we did evaluate the influence of rotor vibrations on the measured energy shift between emission and absorption lines. For this purpose, we carried out identical measurements of the intensity of resonant $\gamma$-radiation passing across the resonant absorber at various rotational frequencies for two different absorbers whose resonant lines are shifted with respect to each other on the energy scale approximately at the linewidth. Thereby, we obtained the possibility to estimate separately the variation of the detector's count rate due to the broadening of the line caused by vibrations and due to the actual energy shift between emission and absorption resonant lines [10, 13]. Having measured this energy shift at different tangential velocities $u$, we got the unbiased estimate of

\footnotetext{
${ }^{1}$ In 2015, after the performance of the experiments [10-13], equation (2) had been corrected to the form [14]

$$
\frac{\Delta E}{E}=-\frac{2}{3} \frac{u^{2}}{c^{2}},
$$

for co-rotating source and absorber, which however did not reduce the motivating role of eq. (2) in our aspiration to re-analyze the Mössbauer rotor experiments.

${ }^{2}$ We present this information in order to deny the opinion, which we faced during the last years, that under the implementation of experiments $[10,11]$ and $[12,13]$ we were allegedly influenced by "confirmation bias" where the researchers unknowingly get led astray by a predilection to confirm a specific prediction.
} 
the coefficient $k$ in eq. (4), though with a larger random component of measurement uncertainty compared to the experiments [1-6].

Finally, we obtained $k=0.66 \pm 0.03[11]$, and $k=0.69 \pm 0.02[12,13])$, which actually signify that the coefficient $k$ in eq. (4) is equal to $2 / 3$.

During the past few years, there were several attempts to explain the result $k=2 / 3$, where, for convenience, its deviation from the classical relativistic prediction $k=1 / 2$ was named as the "extra-energy shift" (EES), i.e.

$$
\left(\frac{\Delta E}{E}\right)_{\text {extra }}=-\frac{1}{6} \frac{u^{2}}{c^{2}}
$$

One of the firsts attempts to explain the origin of the EES has been made by Corda (see, e.g. $[15,16]$, where he claimed the presence of the so-called "synchronization effect" between the clock at the origin of a rotating system and the laboratory clock. However, as we have previously shown in $[17,18]$, this synchronization effect, even if it existed, is totally immeasurable in any Mössbauer rotor experiment. All the same, we have further indicated computational errors by Corda in the derivation of his "synchronization effect" [19], which thus totally invalidate his approach.

One more approach to the interpretation of eq. (5) had been suggested by Friedman et al. $[20,21]$, where the presence of a "maximal acceleration" $a_{m}$ in Nature had been assumed. In such a case, the EES with the value (5) corresponds to the maximal acceleration near $10^{19} \mathrm{~m} / \mathrm{s}$. However, as it was shown in ref. [22] via the analysis of the temperature dependence of the shifts of resonant lines in crystals, the lowest limit of maximal acceleration, if it ever existed, should be near $10^{21} \mathrm{~m} / \mathrm{s}$. With this value of $a_{m}$, the corresponding EES in the Mössbauer rotor experiments becomes miniscule and practically immeasurable.

Further on, we would like to mention recent attempts to explain the EES (5) via the socalled "time dependent Doppler shift" [23], or via the "desynchronization effect" [24], where the erroneous character of both attempts had been disclosed in our papers [25] and [7] respectively.

Finally, Podosenov et al. have published their recent contribution [26], where they claim that the "synchronization effect" à-la Corda should exist, which allegedly explains the origin of the EES.

However, in section 2, we will disclose serious errors in the physical interpretation of the results of [26], which make them inapplicable to any of the Mössbauer experiments in a rotating system conducted up to now. Finally, we conclude in section 3.

\section{Frequency shift of resonant $\gamma$-quanta emitted from the axis of a rotating disc}

The title of the present section directly reflects the goal of the recent paper by Podosenov et al. [26], where the authors conclude that Corda, in his paper [16], “...correctly interprets the Mössbauer experiment in a rotating reference frame...”. We feel deceived that, by making this claim, Podosenov et al. totally missed our recent contribution [19] mentioned above, where we disclosed computational errors by Corda in the derivation of his "synchronization effect" in [15, $16]$.

Moreover, advocating the "synchronization effect" by Corda, Podosenov et al. suggest, in fact, quite a different way to explain the EES in comparison with the approach by Corda [15, 16]. In fact, there is only a single common point in refs. $[15,16,26]-$ the use of the Langevin metric, i.e.

$$
d s^{2}=\left(1-\frac{r^{\prime 2} \omega^{2}}{c^{2}}\right) c^{2} d t^{2}-2 \omega r^{2} d \phi^{\prime} d t^{\prime}-d r^{2}-r^{2} d \phi^{2}-d z^{2}
$$

resulting from the transformation between the inertial frame (non-primed coordinates) and the rotating frame (primed coordinates)

$$
t=t^{\prime}, r=r^{\prime}, \phi=\phi^{\prime}+\omega t^{\prime}, z=z^{\prime},
$$


with $\omega$ being the angular velocity (see, e.g. [27]). Here, we add that the metric (6) has also been used in some other papers devoted to the origin of EES (e.g., [7, 24].

Further on, considering the propagation of the resonant $\gamma$-quanta from the origin of a rotating system to the rotor edge, Podosenov et al. [26] apply the constraint

$$
\phi^{\prime}=\text { const, }
$$

whereas Corda had used the equality $[15,16]$

$$
\phi=\text { const }
$$

which substantially differs in its physical meaning from (8a). Indeed, eq. (8a) signifies that resonant $\gamma$-quanta, having been emitted from the source at the origin, propagate along the radial coordinate $r^{\prime}$ of the rotating frame, whereas the equality (8b) corresponds to the propagation of resonant $\gamma$-quanta along the radial coordinate $r$ of the laboratory (non-rotating) frame.

This observation already indicates that Corda $[15,16]$ and Podosenov et al. [26] considered, in fact, different physical problems.

Next, Podosenov et al. [26] calculate the proper time $\tau_{1}$ of the propagation of light from the origin of the rotating system to the rotor edge $R$ :

$$
\tau_{1}=\frac{R}{c}\left(1+\frac{2 X^{2}}{3}\right),
$$

where $X=\omega R / c$ (see eq. (18) of [26]). Then, they introduce into consideration two short flashes emitted by a radiator at the origin of a rotating disk, which are separated by the time interval $\delta t$. Hence, at the stationary metric (6), one gets

$$
\tau_{1}+\delta \tau_{1}=\left(\frac{R}{c}+d t\right)\left(1+\frac{2 X^{2}}{3}\right) .
$$

Introducing, for repeating periods, the corresponding frequencies $1 / \delta t=\mathrm{v}_{0}, 1 / \delta \tau_{1}=\mathrm{v}_{R}$, the authors of [26] arrive at the equality

$$
v_{R}=v_{0}\left(1-\frac{2 X^{2}}{3}\right),
$$

which, in their opinion, does coincide with the experimental results [10-13] and with the theoretical result [16].

However, it becomes obvious that Podosenov et al. [26] did not even realize the fact that eq. (11) indicates a red shift of the frequency of the resonant radiation (i.e., $v_{R}<v_{0}$ ), whereas the equality $k=2 / 3$ in eq. (4), obtained in the experiments [10-13] corresponds to the blue shift of the resonant radiation when $v_{R}>v_{0}$. We add that the same blue shift of the frequency of the resonant $\gamma$-quanta has been obtained in all other Mössbauer rotor experiments [1-6] in the configuration where the source of resonant radiation was located on the rotational axis, and the resonant absorber was mounted on the rotor rim.

Thus, the equation (11) derived by Podosenov et al. in ref. [26] obviously contradicts the experimental results [1-6, 10-13].

One of the reasons of such a contradiction can be immediately indicated: The constraint (8a) used in ref. [26] implies that the resonant $\gamma$-quanta will propagate along the radial coordinate $r$ ' of the rotating system, and hence, a laboratory observer would see the propagation of such $\gamma$ quanta along a curved path. However, this situation is impossible when $\gamma$-radiation propagates in empty space, just like in all experiments [1-6, 10-13]; thus, the equality (8a) inevitably implies the presence of a suitable photon guide of resonant $\gamma$-radiation rotating with the angular velocity $\omega$, which connects the source on the rotational axis and the absorber on the rotor rim.

Be that as it may, we would like to emphasize that no guides of resonant $\gamma$-quanta had ever been used in the known Mössbauer rotor experiments (including [1-6, 10-13]). Moreover, such a guide is not even invented yet. Therefore, the analysis of Podosenov et al. [26], based on eq. (8a), is totally inapplicable to any of the experiments mentioned above $[1-6,10-13]$, where $\gamma$ - 
quanta emitted by a spinning source mounted on the rotor freely propagate along straight lines as seen by the laboratory observer. For example, in the configuration of Fig. 1, this straight line joins the source and the detector. It is obvious that, for resonant $\gamma$-quanta propagating in empty space, eq. (8a) used by Podosenov et. al in [26] must be replaced by eq. (8b).

With these explanations, the physical meaning of all results derived in ref. [26] become easily understandable for a laboratory observer if one takes into account that, for this observer, the constraints (8a) means that resonant $\gamma$-quanta inside a rotating guide propagate along a curved path. Then, it is not surprising that the time of their propagation from the source to the absorber is given by eq. (9), which happens to be larger than $R / c$.

The latter result explains, in particular, the emergence of the frequency shift in eq. (11), which, however, still does not describe the total frequency (energy) shift of the resonant line for a resonant absorber. Namely, one has to additionally take into account the time dilation effect in the rotating resonant absorber with the inclusion of the EES, which, according to the experimental results [10-13], has the form

$$
v_{a}=v_{R}\left(1+\frac{2 X^{2}}{3}\right) .
$$

Here, we have used the designations of ref. [26], and denoted via $v_{a}$ the frequency of the resonant radiation for an observer attached to the absorber. Further, substituting eq. (11) into eq. (12), we finally obtain

$$
v_{a}=v_{0}\left(1-\frac{2 X^{2}}{3}\right)\left(1+\frac{2 X^{2}}{3}\right) \approx v_{0}
$$

where we drop the terms in orders of magnitude higher than $c^{-2}$.

Thus, the correct completion of the analysis of [26] allows us to conclude that, in the case where the resonant $\gamma$-quanta propagate inside a special guide, by connecting the co-rotating source situated on the rotational axis to the absorber on the rotor rim, the measured position of the resonant line of the orbiting absorber remains unshifted on the energy (frequency) scale, which is explained by the mutual counterbalancing of the effects (11) and (12).

At the same time, in the absence of such a guide of resonant $\gamma$-radiation (which still remains hypothetical at the moment), we have to replace eq. (8a) used in ref. [26] by eq. (8b) corresponding to the free propagation of the resonant radiation from a source to an absorber. In this case, combining eqs. ( $8 \mathrm{~b})$ and $(7 \mathrm{c})$, we get the equality

$$
\phi^{\prime}=-\omega t^{\prime} .
$$

Further substitution of eq. (14) into the Lengevin metric (6) yields

$$
d s^{2}=c^{2} d t^{2}-d r^{2}-d z^{2} \text {. }
$$

Considering the rotation in the $x y$-plane (where $d z^{\prime}=0$ ), and putting $d s=0$ for $\gamma$-quanta propagating in empty space, we arrive at the trivial equality

$$
\frac{d r^{\prime}}{d t^{\prime}}=\frac{d r}{d t}=c,
$$

which leaves no room for any "synchronization" effect $[15,16]$, or similarly, any "desynchronization" effect [24], and just indicates that, for a laboratory observer, the resonant $\gamma$ quanta propagate in the radial direction at the light speed $c$. In summary, they travel in straight lines upon emission as seen in the laboratory.

We must add that Corda also used the correct equation (8b) for $\gamma$-radiation propagating from the source to absorber in $[15,16]$. However, in the metric expression (6), he inadvertently neglected some of the terms of the order $c^{-2}$ (which is already inadmissible in the analysis of Mössbauer rotor experiments where the energy shift between emission and absorption lines has the same order of magnitude) and, moreover, used the wrong sign in eq. (14) (i.e., he improperly adopted $\phi^{\prime}=\omega t^{\prime}$ ) - whereby, these errors totally invalidate his "synchronization effect" (for more details, see [19]). 
In the proper approach, the constraint (8b) straightforwardly yields equation (16), which should anyway be taken into account in all further attempts to interpret the origin of the EES under the framework of the general theory of relativity.

\section{Conclusion}

In the present contribution, we have shown the failure of a newer attempt [26] to explain the origin of the EES in Mössbauer rotor experiments. This is first of all due to the fact that the experimental configuration considered in [26] implies the presence of a special guide for resonant $\gamma$-quanta to ensure eq. (8a), which however has never been realized, and could not have been realized in all known experiments on this subject [1-6, 10-13] because such guides are not invented yet!

Nevertheless, if guides for resonant $\gamma$-radiation were invented in the near future, then the results reported in [26] could be useful in the calculation of the energy shift between emission and absorption resonant lines when such a guide is used. In particular, under the natural assumption that the observed count rate of the detector of resonant $\gamma$-radiation totally originates from the relative energy shift between emission and absorption resonant lines, the measured detector's count rate should remain unchanged at different rotational frequencies (see eq. (13)) due to the mutual counterbalancing of the effects (11) and (12). This result, if confirmed, definitely could shed more light on the essence of the EES, which at the moment remains unexplained under the framework of the general theory of relativity.

\section{References}

1. H.J. Hay et al. Phys. Rev. Lett. 4, 165 (1960).

2. D.C. Champeney, P.B. Moon. Proc. Phys. Soc. 77, 350 (1961).

3. H.J. Hay. In: Proc. of Second Conference on the Mössbauer effect, ed. by A. Schoen and D.M.T Compton (Wiley, New York, 1963), p. 225.

4. T.E. Granshaw, H J. Hay. In: Proc. Int. School of Physics, "Enrico Fermi" (Academic Press, New York, 1963), p. 220.

5. W. Kündig. Phys. Rev. 129, 2371 (1963).

6. D.C. Champeney, G.R. Isaak, A.M. Khan. Proc. Phys. Soc. 85, 83 (1965).

7. A.L. Kholmetskii, T. Yarman, O. Yarman, M. Arik, Ann. Phys. 411, 167912 (2019).

8. T. Yarman. Ann. Fond. de Broglie 29, 459 (2004).

9. A.L. Kholmetskii, T. Yarman and O.V. Missevitch, Phys. Scr. 78, 035302 (2008).

10. A.L. Kholmetskii, T. Yarman, O.V. Missevitch, B.I. Rogozev, Phys. Scr. 79, 065007 (2009).

11. A.L. Kholmetskii, T. Yarman, O.V. Missevitch, Int. J. Phys. Sci. 6, 84 (2011).

12. A.L. Kholmetskii, T. Yarman, M. Arik and O.V. Missevitch, AIP Conf. Proc. 1648, 510011 (2015).

13. T. Yarman, A.L. Kholmetskii, M. Arik, B. Akkus, Y. Öktem, L.A. Susam, O.V. Missevitch, Can. J. Phys. 94, 780

14. T. Yarman, A.L. Kholmetskii, M. Arik, Eur. Phys. J. Plus 130, 191 (2015).

15. C. Corda, Ann. Phys. 355, 360 (2015).

16. C. Corda, Ann. Phys. 368, 258 (2016).

17. A.L. Kholmetskii, T. Yarman, M. Arik, Ann. Phys. 363, 556 (2015).

18. A.L. Kholmetskii, T. Yarman, O. Yarman, M. Arik, Ann. Phys. 374, 247 (2016).

19. A.L. Kholmetskii, T. Yarman, O. Yarman, M. Arik, Ann. Phys. 409, 167931 (2019).

20. Y. Friedman and Yu. Gofman, Phys. Scr. 82, 015004 (2010).

21. Y. Friedman, Ann. der Phys. 523, 408 (2011).

22. W. Potzel, Hyperfine Interact. 237, 38 (2016).

23. E. Benedetto and A. Feoli, Eur. Phys. J. Plus 133, 53 (2018).

24. G. Iovane and E. Benedetto. Ann. Phys., 403, 106 (2019).

25. A.L. Kholmetskii, T. Yarman, O. Yarman, M. Arik, Eur. Phys. J. Plus 133, 261 (2018). 
26. S.A. Podosenov, J. Foukson, E.R. Men'kova, Ann. Phys., in press; doi.org/10.1016/j.aop.2019.168047.

27. L. Landau and E. Lifshits, Classical Theory of Fields (3rd ed.) (London, Pergamon 1971). 


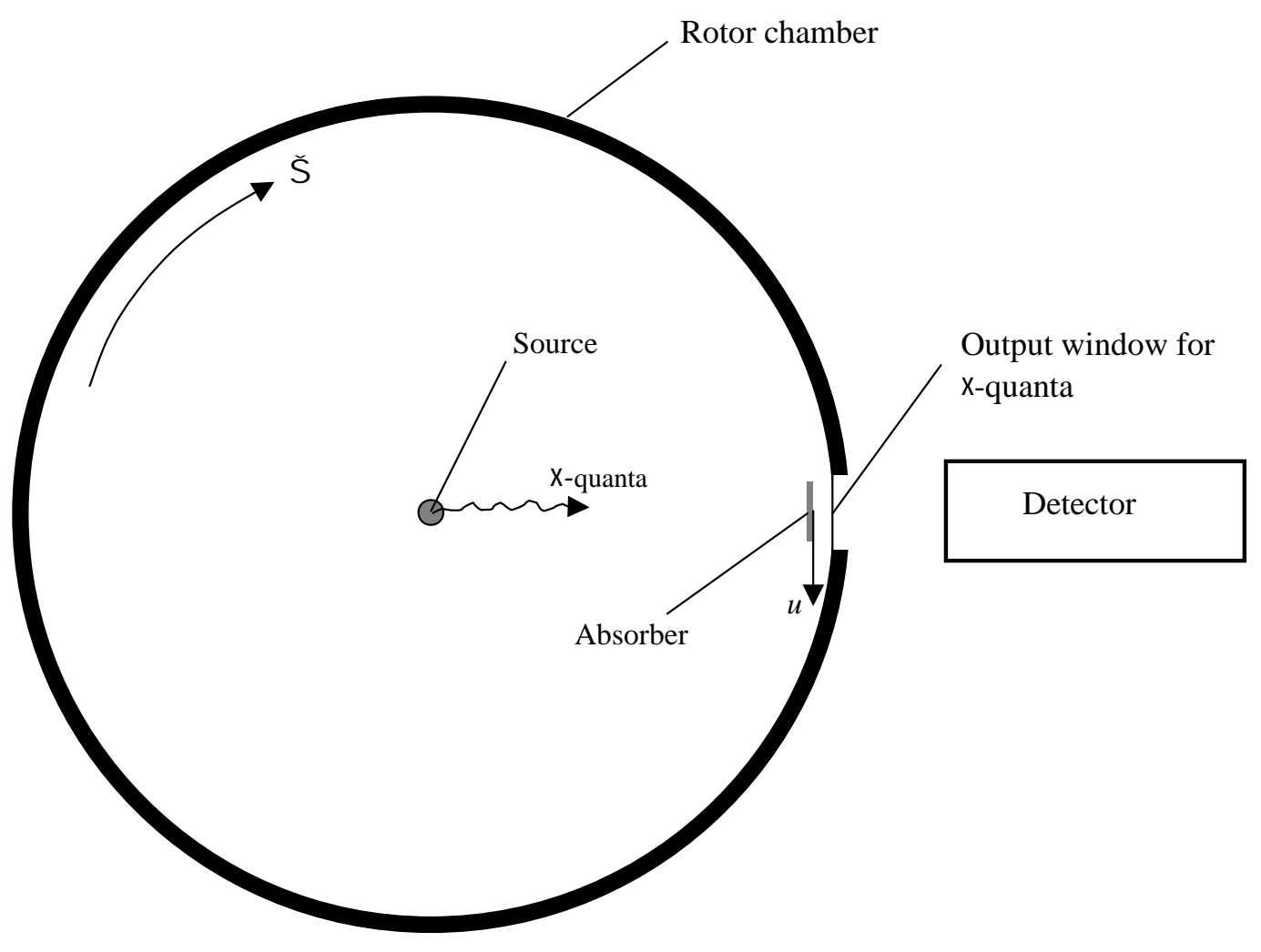

Figure 1 adapted from [7]: Sketch of the Mössbauer rotor experiment. A source of resonant radiation is mounted on the rotor axis; an absorber is located on the rotor rim, while a detector of $\gamma$-quanta is placed outside the rotor system. $\gamma$-quanta emitted from the source and passing across the absorber are detected at the time moments when the source, absorber and detector are aligned in a straight line. 